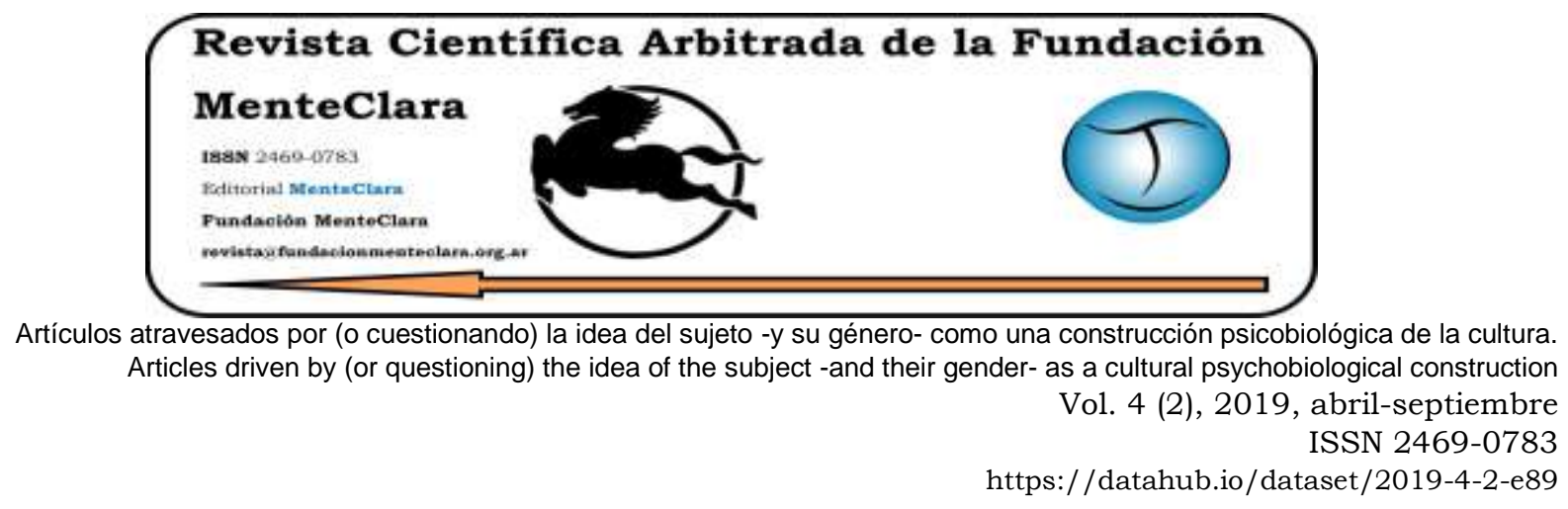

\title{
RELIGIOSIDAD Y ESPIRITUALIDAD EN EL MARCO DEL MODELO DE LOS CINCO FACTORES DE LA PERSONALIDAD
}

\author{
RELIGION AND SPIRITUALITY WITHIN THE FIVE-FACTOR MODEL OF \\ PERSONALITY
}

Hugo Simkin¹, Pablo Rubio², Gabriela Di Puglia ${ }^{3}$, Maximiliano Preuss ${ }^{4}$

Cómo citar este artículo / Citation: Simkin H., Rubio P., Di Puglia G., Preuss M. (2019). "Religiosidad y espiritualidad en el marco del modelo de los cinco factores de la personalidad". Revista Científica Arbitrada de la Fundación MenteClara, 4(2) abrilseptiembre 2019, 73-109.

DOI: https://doi.org/10.32351/rca.v4.2.89

Copyright: (C) 2019 RCAFMC. Este artículo de acceso abierto es distribuido bajo los términos de la licencia Creative Commons Attribution 4.0 International License (CC BY 4.0). Recibido: 06/07/2019. Aceptado: 11/07/2019 Publicación online: 30/10/2019

Conflict of interests: Ninguno que declarar.

\section{Resumen}

A pesar de que el vínculo entre la espiritualidad y los rasgos de la personalidad fue intensamente indagado en la literatura académica en los últimos años particularmente en el marco del modelo y la teoría de los cinco factores de la personalidad, no existen trabajos de revisión que permitan sintetizar el estado del arte respecto de esta relación. Por este motivo, el propósito del presente trabajo es revisar los estudios que se proponen indagar los constructos numinosos en atención a las diferencias individuales a partir de una revisión sistemática de la bibliografía en las bases de datos PsycInfo, ERIC, Pubmed, CAIRN, CLASE, Scielo, Dialnet, Lilacs y Redalyc. De acuerdo con la revisión, si bien existe una considerable disparidad de

\footnotetext{
${ }^{1}$ Universidad de Buenos Aires, UBA. hugosimkin@psi.uba.ar

2 Universidad de Flores, UFLO.

3 Universidad Abierta Interamericana, UAI.

4 Universidad Abierta Interamericana, UAI.
} 
resultados, generalmente se han reportado asociaciones positivas entre estos constructos y la apertura, la responsabilidad, la extroversión, y la amabilidad, y negativas con el neuroiticismo. Se concluye que la discrepancia en los resultados respondería a la multiplicidad de técnicas de evaluación disímiles empleadas para relevar la espiritualidad y la religiosidad.

\begin{abstract}
Although the link between spirituality and personality traits has been intensely researched in the academic literature in recent years -particularly within the fivefactor personality model and theory-, there are no review articles that allow summarizing the state of the art in such relationship. Thus, the purpose of this paper is to review studies analyzing the numinous constructs related to individual differences by means of a systematic review of the bibliography found in the PsycInfo, ERIC, Pubmed, CAIRN, CLASS, Scielo, Dialnet, Lilacs, and Redalyc databases. According to such review, although results show considerable disparity, there have been reported positive associations between said constructs and openness, responsibility, extroversion, and agreeableness as well as negative associations with neuroiticism. It is concluded that the discrepancy in the results would be due to to the multiplicity of dissimilar psychological techniques used to assess spirituality and religiosity.
\end{abstract}

Palabras Claves: Personalidad; espiritualidad; $\mathrm{MCF}$

Keywords: Personality; spirituality; FFM 


\section{Introducción}

La psicología de la personalidad se ha interesado tempranamente en las variables numinosas. Desde sus orígenes, distintos autores han debatido si estos constructos representan tendencias básicas de la personalidad, o si se trata de características adaptativas.

El presente artículo se propone relevar los principales antecedentes en psicología de la personalidad y en psicología de la espiritualidad y de la religión que han procurado establecer un vínculo entre los constructos numinosos y las diferencias individuales en el marco del modelo de los cinco factores de la personalidad.

Si bien son numerosos los estudios empíricos que han dado cuenta de dicha asociación, resultan escasos los trabajos que se ocupan de sistematizar los distintos enfoques relativos a su relación teórica.

Por este motivo, en la presente revisión se sigue principalmente el trabajo The Role of Personality in Understanding Religious and Spiritual Constructs, publicado por Ralph Piedmont en la primera edición del Handbook of Religion and Spirituality editado por Raymond Paloutzian y Crystal Park en 2005 y su revisión en la edición de 2012 publicado junto a Theresa Wilkins.

Finalmente, se siguen los desarrollos de Ralph Piedmont relativos a las diferencias entre la religiosidad, la espiritualidad y el FFM, su lugar estructural dentro del modelo, y el carácter universal de la espiritualidad.

\section{Religiosidad y espiritualidad en psicologia de la personalidad}

Desde sus inicios, la espiritualidad y la religiosidad han resultado de gran interés en el área de la psicología de la personalidad, dado que se han encontrado fuertes asociaciones entre la búsqueda de lo sagrado y 
determinados patrones de pensamientos, emociones y comportamientos (Argyle \& Beit-Hallahmi, 1975; Emmons, 1988, 1999a; Piedmont \& Wilkins, 2013; Robbins \& Francis, 2000).

Sin embargo, a partir del dossier editado por Robert A. Emmons en la revista Journal of Personality sobre religión en psicología de la personalidad a fines de la década del noventa, los artículos que vinculan estas variables crecieron exponencialmente (Emmons, 1999b; Rose \& Exline, 2012).

A partir de estos trabajos, con diferentes matices (Piedmont, 1999b; Saroglou \& Fiasse, 2003; Saroglou \& Muñoz García, 2008), la mayoría de los estudios han observado que la personalidad puede contribuir a que un individuo se encuentre cómodo participando de actividades espirituales o religiosas, a la vez que ciertos contextos religiosos pueden incidir en el desarrollo de la personalidad a lo largo de la socialización (Koenig, King, \& Carson, 2012; McCullough \& Worthington, 1999), tal como sugieren diversas revisiones (Sanua, 1969) y estudios meta analiticos (Lodi-Smith \& Roberts, 2007).

\section{Cinco teorias de la personalidad}

Si bien existen diversas teorias para explicar el vínculo entre los constructos numinosos y las diferencias individuales (Francis \& Bourke, 2003; Kirkpatrick, 1999; Walborn, 2014), Piedmont (2005) identifica cinco modelos de la personalidad que son frecuentemente estudiados conjuntamente con la religiosidad y la espiritualidad:

Por una parte la teoría de la relación de objetos y el estilo de apego, que representan teorias de alcance intermedio que por lo general se enfocan en fenómenos psicológicos particulares; y por otra, la tipología 
de Eysenck, el modelo biopsicosocial de Cloninger y el FFM, que representan modelos más generales de la personalidad.

En particular, diferentes autores han observado que el FFM representa uno de los modelos de mayor relevancia, dado que permite conocer el desarrollo y expresión de los constructos numinosos a lo largo del ciclo vital, su importancia adaptativa, y el modo en que se asocian a las diferencias individuales (Ashton \& Lee, 2014; Chang et al., 2015; Piedmont, Ciarrocchi, Dy-Liacco, \& Williams, 2009; Piedmont \& Wilkins, 2013; Rose \& Exline, 2012).

En este sentido, Ozer y Reise (1994) sostienen que asociar un constructo al FFM resulta equivalente a establecer la latitud y longitud de una determinada locación en el mapa del planeta Tierra.

A partir de esta premisa, Piedmont (2005) sugiere que continuar evaluando la religiosidad y espiritualidad sin localizarlas en el FFM solo puede compararse con la actitud de un geógrafo que reporta una nueva tierra, pero se rehúsa a localizarla en un mapa.

\section{El modelo de los cinco factores}

Distintos autores han observado que el FFM juega un rol importante en el estudio de la religiosidad y de la espiritualidad, dado que permite conocer su desarrollo y expresión a lo largo del ciclo vital, su importancia adaptativa y el modo en que se asocian a las diferencias individuales (Ashton \& Lee, 2014; Chang et al., 2015; Piedmont, Ciarrocchi, et al., 2009; Piedmont \& Wilkins, 2013; Rose \& Exline, 2012; Saroglou \& Jaspard, 2000).

Además, Piedmont (2005), señala que el FFM también provee un modo de asociar los constructos religiosos entre sí. Así, aquellas escalas de 
religiosidad o de espiritualidad que se relacionen de manera similar al FFM, deberian compartir mucho personológicamente, mientras que diferentes patrones correlacionales podrian indicar que las dos escalas tienen muy poco en común (Piedmont, 2005).

Desde esta perspectiva, distintos estudios han encontrado relaciones entre los constructos numinosos y el FFM en diferentes países como Australia (Browne, Pennycook, Goodwin, \& McHenry, 2014), Austria (Schnell, 2012), Bélgica (Duriez \& Soenens, 2006; Duriez, Soenens, \& Beyers, 2004; Saroglou \& Fiasse, 2003), Canadá (Browne et al., 2014; Taylor \& MacDonald, 1999), España (Saroglou \& Muñoz García, 2008), Eslovaquia (Gajdosova, Orosova, Janovska, \& Benka, 2014; Halama, Martos, \& Adamovová, 2010), Estados Unidos (Henningsgaard \& Arnau, 2008; Rowatt \& Kirkpatrick, 2002), Hungria (Halama et al., 2010), Irán (Aghababaei, 2013; Aguilar-Vafaie \& Moghanloo, 2008; Mirsaleh, Rezai, Kivi, \& Ghorbani, 2010; Salmanpour \& Issazadegan, 2012), Inglaterra (Lewis, 1999), Noruega (Kaldestad, 1996) o Turquía (Dirilen-Gümüş, 2010), en diferentes religiones (Aghababaei, 2013; Aguilar-Vafaie \& Moghanloo, 2008) y grupos etarios (Taylor \& MacDonald, 1999).

Si bien la mayoría de los diseños son transversales, también es posible identificar distintos estudios longitudinales (Heaven \& Ciarrochi, 2007; McCullough, Enders, Brion, \& Jain, 2005; McCullough, Tsang, \& Brion, 2003; Wink, Ciciolla, Dillon, \& Tracy, 2007).

Además, siguiendo las recomendaciones de Saroglou, Pichon, Trompette, Verschueren y Dernelle (2005), diferentes autores han empleado la perspectiva del otro observador (Dillon, Wink, \& Fay, 2003; Saroglou \& Fiasse, 2003; Wink et al., 2007) y diseños experimentales (Ahmed, 2009; Ahmed \& Salas, 2013). 
En una temprana revisión del estado del arte, Koenig (1998) identifica distintos estudios pioneros en explorar las relaciones entre la religiosidad, la espiritualidad y las diferencias individuales.

Entre los primeros estudios se encuentra el trabajo de Costa, McCrae y Norris (1981), quienes concluyen que la religiosidad se encuentra inversamente asociada al neuroticismo y positivamente a la introversión.

Posteriormente, Koenig et al., (1990) encuentran que la religiosidad promueve un aumento en la estabilidad emocional a lo largo del tiempo. En un estudio meta-analitico que incluye 71 muestras de diferentes continentes como Asia, Europa y Oceania, Saroglou (2010) identifica tres categorias generales a partir de las cuales es posible clasificar la multiplicidad de escalas empleadas para evaluar los constructos numinosos que estos estudios relacionan al FFM:

(1) la religiosidad, que define como el conjunto de creencias y prácticas vinculadas a lo trascendente legitimadas por un grupo o tradición,

(2) la espiritualidad, que caracteriza como el vínculo con una realidad trascendente, pero fuera del marco de las instituciones o tradiciones formales y

(3) el fundamentalismo religioso, que identifica a aquellas personas que presentan una visión dogmática de creencias, prácticas y actitudes ligadas a lo trascendente.

De acuerdo con el autor, las tres variables reflejan correlaciones significativas con responsabilidad y amabilidad, espiritualidad particularmente se asocia a extroversión, mientras que apertura se relaciona positivamente con espiritualidad, pero negativamente con fundamentalismo y no se asocia a la religiosidad. 
En una revisión reciente, Koenig et al., (2012) han relevado numerosos estudios publicados desde el año 2000 que evalúan las relaciones entre los constructos numinosos y los rasgos de la personalidad.

Los autores identifican 54 estudios que encuentran asociaciones entre la religiosidad y el neuroticismo, de los cuales el $24 \%$ reportan una relación negativa, el $9 \%$ una relación positiva y el $61 \%$ ninguna asociación, 50 estudios que la asocian a la extraversión, de los cuales el $38 \%$ encuentra una relación positiva, el 6\% una negativa y el 54\% ninguna relación, 30 estudios que la asocian a la responsabilidad, de los cuales el $63 \%$ encuentra una relación positiva, 3\% negativa y el 30\% ninguna relación, 30 estudios que la asocian a la amabilidad, de los cuales el $87 \%$ se asocia positivamente, $0 \%$ negativamente y $7 \%$ ninguna asociación, y 26 estudios que la vinculan a la apertura, de los cuales el $42 \%$ encuentra una relación positiva, el 12\% una relación negativa y $38 \%$ ninguna relación.

Siguiendo a Saroglou (2002), los autores sostienen que si se controla espiritualidad, es probable que la relación tienda a ser negativa $(r=-.14)$.

Tabla 1 - Asociaciones entre la religiosidad/espiritualidad y los cinco factores desde el año 2000

\begin{tabular}{lcccc}
\hline & Positiva & Negativa & Ninguna asociación & N Estudios \\
\hline 1. Apertura & $42 \%$ & $12 \%$ & $38 \%$ & 26 \\
2. Responsabilidad & $63 \%$ & $3 \%$ & $30 \%$ & 30 \\
3. Extraversión & $38 \%$ & $6 \%$ & $54 \%$ & 50 \\
4. Amabilidad & $87 \%$ & $0 \%$ & $7 \%$ & 30 \\
5. Neuroticismo & $9 \%$ & $24 \%$ & $61 \%$ & 54 \\
\hline Adaptado & & &
\end{tabular}

Adaptado de Koenig., et al. (2012) 


\section{Espiritualidad, religiosidad y apertura}

Diferentes autores han explorado el vinculo entre la espiritualidad, la religiosidad y la apertura a la experiencia a partir de la teoría de los rasgos (Duriez et al., 2004; Saucier \& Skrzypińska, 2006; Village, 2011). En líneas generales, la espiritualidad ha sido asociada a la mayoría de las facetas de la apertura (García \& Saroglou, 2008; Koenig et al., 2012; Saroglou \& Fiasse, 2003).

De acuerdo con la literatura, el interés por conocer lo diferente, característico de este factor, podría suponer a la vez una motivación por explorar las experiencias trascendentes (Piedmont, 1999b, 2012).

Por su parte, si bien en numerosos estudios la relación entre la religiosidad y la apertura también puede parecer positiva, si se controla la espiritualidad, esta tiende a resultar negativa (Browne et al., 2014; McCullough \& Willoughby, 2009; Saroglou, 2002).

Por este motivo, diferentes autores consideran que la religiosidad y la apertura deberian asociarse de manera inversa (Roberts \& Robins, 2000; Saucier \& Skrzypińska, 2006).

No obstante, aunque son pocos los estudios que distinguen las diferentes facetas del factor (Rose \& Exline, 2012), aquellos estudios en que han sido discriminadas señalan que, aunque la religión no parece encontrarse asociada a la apertura a los valores, sí parece relacionarse con apertura a las ideas, a la estética o a los sentimientos (Saroglou \& Fiasse, 2003; Saroglou \& Muñoz García, 2008).

Estos resultados parecen indicar que no todos los aspectos de la religiosidad se asocian a una baja apertura, y a otros constructos relacionados, como el dogmatismo, el autoritarismo o el fundamentalismo religioso, tal como han sugerido numerosos 
antecedentes (Duck \& Hunsberger, 1999; Feagin, 1964; García \& Saroglou, 2008; Jonathan, 2008).

Así, los estudios que incluyen la Escala de Orientación Religiosa de Búsqueda (Batson, 1976) o la Escala de Creencias Post-Criticas (Duriez, Fontaine, Hutsebaut, \& Leuven, 2000), que permiten relevar características ecuménicas de las experiencias religiosas, tienden a encontrar asociaciones positivas con apertura y negativas con el fundamentalismo religioso y con el prejuicio (Hunsberger, 1995; Saroglou \& Jaspard, 2000; Whitley, 2009).

Esto podría deberse a la tendencia de los fundamentalistas religiosos a repudiar ciertos aspectos del mundo moderno, apegándose a doctrinas religiosas conservadoras (Saroglou \& Muñoz García, 2008; Streyffeler \& McNally, 1998).

\section{Espiritualidad, religiosidad y responsabilidad}

Distintos estudios meta-analiticos han identificado una moderada relación entre la responsabilidad y la religiosidad (Lodi-Smith \& Roberts, 2007; Saroglou, 2002).

En parte, tal vínculo podría deberse a que el autocontrol y la moral, aspectos usualmente asociados al factor responsabilidad, resultan centrales para la mayoría de las religiones (Aghababaei, 2013; Baumeister \& Exline, 1999; Rose \& Exline, 2012).

De acuerdo con Rose y Exline (2012), estas características podrian contribuir a explicar además la asociación inversa encontrada entre la religiosidad y variables frecuentemente asociadas a la impulsividad, como la adicción a las drogas. 
Sin embargo, tal como sucede en otros casos, la relación moderada podría ser consecuencia de la considerable disparidad en las técnicas de evaluación empleadas, de modo que distintos aspectos de la religiosidad podrían asociarse a la responsabilidad de manera independiente (Rose $\&$ Exline, 2012).

Por ejemplo, la internalización de los preceptos morales que promueven las religiones podría variar en función de la orientación religiosa intrínseca o extrínseca de las personas, de modo que aquellos individuos más comprometidos con sus creencias tendrian una mayor tendencia a respetar los preceptos morales, mientras que aquellas personas cuya relación con las instituciones se encuentra motivada por los beneficios sociales obtenidos solo aparentarian dicha moralidad (Aquino \& Reed, 2002; Carpenter \& Marshall, 2009; Maclean, Walker, \& Matsuba, 2004).

Con relación a la espiritualidad, se ha señalado que son relativamente escasos los estudios que han procurado distinguir el vínculo con la responsabilidad con independencia de la religiosidad, por lo que se han reportado resultados contradictorios (García \& Saroglou, 2008; McCullough \& Willoughby, 2009; Rose \& Exline, 2012).

Si bien es cierto que la espiritualidad presenta un menor apego por las normas, tradiciones y rituales que la religiosidad, siguiendo a Piedmont (1999a), esta se caracteriza por una sensación de responsabilidad con la humanidad.

Desde este enfoque, se ha destacado que las facetas de la espiritualidad relevadas por la Escala de Evaluación de Espiritualidad y Sentimientos Religiosos se han asociado positivamente a la responsabilidad (Chang et al., 2015; Piedmont, 2012). 


\section{Espiritualidad, religiosidad y extraversión}

Entre los primeros estudios que han vinculado estas variables, Francis, Pearson y Kay (1983) señalaron que la religiosidad se asocia a la introversión debido a que numerosas características de este factor suelen valorarse de manera positiva en diferentes religiones.

Desde entonces, si bien los resultados de numerosos trabajos que encuentran relación entre estos constructos señalan que ésta resulta positiva, en la actualidad un amplio porcentaje de estudios concluyen que estas variables no se encuentran relacionadas (Francis \& Pearson, 1985; Francis et al., 1983; Koenig et al., 2012).

Una vez más, se observa que las diferencias en los resultados puede depender de las técnicas de evaluación empleadas (Hill, 2012; Kapuscinski \& Masters, 2010).

La orientación religiosa extrínseca, tal como es evaluada por la Age Universal, posiblemente permita identificar un tipo de religiosidad propio de aquellas personas con mayor extraversión (Donahue, 1985; Kaldestad, 1995; Saroglou, 2002).

Por su parte, el modelo circumplejo de la espiritualidad (Piedmont, 2004b) podría contribuir a discriminar una espiritualidad ascética, ligada a una mayor introversión, de una espiritualidad transpersonal-relacional vinculada a una mayor extraversión.

\section{Espiritualidad, religiosidad y amabilidad}

La mayoria de las revisiones y de los estudios meta-analiticos han reportado una relación moderada entre las facetas de la amabilidad y de la religiosidad (Costa, McCrae, \& Dye, 1991; García \& Saroglou, 2008; Lodi-Smith \& Roberts, 2007; Saroglou, 2002). 
El vínculo podría explicarse debido a que la amabilidad y el comportamiento prosocial son características que promueven la mayoría de las religiones (Rose \& Exline, 2012; Saroglou, 2010).

Además, dado que la amabilidad se caracteriza por el intento de mantener la armonía en los vínculos interpersonales, este factor podría contribuir a disminuir las probabilidades de abandonar las creencias religiosas para evitar el conflicto con los núcleos familiares o las comunidades de pertenencia (Ashton \& Lee, 2014).

Sin embargo, diferentes autores han observado que esta asociación es apenas moderada y, al igual que ocurre en otros casos, puede depender del tipo de religiosidad que se evalúe (Duriez \& Soenens, 2006; Leak \& Fish, 1999; McCullough et al., 2005; Rose \& Exline, 2012).

Por ejemplo, mientras que se ha reportado una mayor amabilidad asociada al compromiso con la religión, y la internalización de los valores religiosos, esta no parece asociarse con la afiliación religiosa (Duriez, 2004; Fontaine, Luyten, \& Corveleyn, 2000; Lodi-Smith \& Roberts, 2007; Streyffeler \& McNally, 1998).

Por otra parte, Exline y Jones (2012) señalan que el vínculo también puede depender de los instrumentos empleados para evaluar la amabilidad.

Esto se debe a que algunas de las facetas se encuentran mejor representadas en unos instrumentos que en otros (Saucier \& Skrzypińska, 2006).

Además de las diferencias en las técnicas empleadas, Rose y Exline (2012) observan dos limitaciones fundamentales de la amabilidad y del comportamiento prosocial en población religiosa. 
En primer lugar, si bien las personas religiosas pueden ser amables en términos generales, en algunos contextos religiosos también son capaces de justificar el uso de la agresión y de la violencia para situaciones específicas (Cota-McKinley, Woody, \& Bell, 2001; Greer, Berman, Varan, Bobrycki, \& Watson, 2005; Leach, Berman, \& Eubanks, 2008).

En particular, se observa que esta justificación para el ejercicio de la violencia puede deberse tanto al tipo de religiosidad, como al contexto social, o al modo en que se interpreten los diferentes textos religiosos (Bushman, Ridge, Das, Key, \& Busath, 2007; Rose \& Exline, 2012).

En segundo lugar, la amabilidad y el comportamiento prosocial pueden quedar restringidos al interior del endogrupo, de modo que mientras que las personas pueden tener un comportamiento de ayuda hacia el interior de la comunidad, experimentarian cierta indiferencia en relación a otros grupos sociales (Batson, Denton, \& Vollmecke, 2008; Batson, Eidelman, Higley, \& Russell, 2001; Batson, Floyd, Meyer, \& Winner, 1999).

Por su parte, los estudios que exploran la relación entre la espiritualidad y la amabilidad, en su mayoria, reflejan asociaciones positivas, de modo que las personas espirituales tienden a valorar más la benevolencia, la empatía y el altruismo y a estar menos interesadas en obtener poder (Henningsgaard \& Arnau, 2008; Leach et al., 2008; MacDonald, 2000; Saroglou et al., 2005).

Sin embargo, el estudio longitudinal de Dillon, Wink y Fay (2003) encuentra que la espiritualidad es predictora de la baja generosidad en la vida adulta.

Tal como señalan Rose y Exline (2012), estos resultados abren una serie de interrogantes, porque contradicen la literatura en el área. 
Posiblemente, el modelo circumplejo propuesto por Piedmont (2004b) pueda contribuir interpretar esta disparidad en los estudios, en tanto permite clasificar una espiritualidad ascética, desvinculada de las relaciones interpersonales y sociales de una espiritualidad transpersonal social, desarrollada en un contexto comunitario.

\section{Espiritualidad, religiosidad y neuroticismo}

Tal como se ha observado, el neuroticismo ha sido definido como la tendencia a experimentar emociones negativas como miedos, sentimientos de culpa, tristeza o enojo (McCrae \& Costa, 2008).

Las personas con mayor neuriticismo suelen presentar creencias irracionales, menor control de los impulsos y una baja capacidad para cooperar con otros (Salmanpour \& Issazadegan, 2012).

En la revisión llevada a cabo por Koenig, King y Carson (2012), el 24\% de los estudios que exploran la relación entre estos constructos reportan una relación negativa, el $9 \%$ una relación positiva y el $61 \%$ ninguna asociación.

Nuevamente, las diferencias entre los resultados pueden depender del modo en que los diferentes constructos fueran evaluados (Hill, 2012; Kapuscinski \& Masters, 2010).

Por un lado, la espiritualidad afecta las elecciones de vida de las personas (Greenberg, Solomon, \& Pyszczynski, 1997), promoviendo un propósito para la existencia (Piedmont, 2004a).

Por su parte, la religiosidad puede ofrecer apoyo social (Salsman, Brown, Brechting, \& Carlson, 2005; Willoughby, Cadigan, Burchinal, \& Skinner, 2008) y estrategias de afrontamiento religiosas que se asocian a una mayor estabilidad emocional (Ahles, Mezulis, \& Hudson, 2016; 
Koenig et al., 1990; Salmanpour \& Issazadegan, 2012; Schnell, 2012). Sin embargo, diferentes estudios han identificado relaciones entre el neuroticismo y una mayor frecuencia en la experimentación de crisis religiosas, en las que las personas perciben e interpretan los diferentes estresores vitales como castigos de Dios o de su comunidad religiosa (Braganza \& Piedmont, 2015; Piedmont, 2012).

Tal como se ha sugerido, la religiosidad podria comprenderse como un modelo mental genérico que puede resultar tanto adaptativo como desadaptativo en el afrontamiento de los diferentes estresores (James \& Wells, 2003; Maltby et al., 2010).

De esta manera, el neuroticismo se encontraría asociado a un modelo mental religioso más desadaptativo, mientras que la estabilidad emocional se vincularía con un modelo mental religioso más adaptativo (Maltby et al., 2010; Simkin \& Azzollini, 2015).

\section{Diferencias entre la espiritualidad y el modelo de los cinco factores}

De acuerdo con Piedmont (1999a), si bien existen similitudes entre la trascendencia espiritual y algunos de los factores del FFM, como la extraversión, la apertura o la amabilidad, el constructo incluye además rasgos de soledad, simplicidad y desapego, características opuestas a estas dimensiones.

Por este motivo, en virtud de que no existe en el FFM un perfil que permita identificar a una persona con alta trascendencia, se ha sugerido que esta podría considerarse como una sexta dimensión del modelo, independiente de los otros cinco factores (Piedmont, 1999a). 
Siguiendo a Piedmont (1999a), como una sexta dimensión de la personalidad, la trascendencia puede contribuir a comprender el modo en que interactuamos con nosotros mismos y con otros, ayudar a reinterpretar nuestras percepciones del ambiente y redefinir los objetivos que perseguimos.

Para el autor, conceptualizar la trascendencia como una dimensión independiente de los otros cinco factores explicaría por qué los individuos persiguen metas trascendentes en contextos tan diversos y por qué las personas con alta trascendencia son tan diferentes entre sí, desde aquellos que glorifican una imagen teística en particular, hasta quienes evitan las religiones formales, creyendo en conceptos amplios, generales, ambiguos y polisémicos como "consciencia elevada".

Finalmente, una característica que permite distinguir la trascendencia de los otros cinco factores de la personalidad es su desarrollo evolutivo (Piedmont, 1999a, 2005).

Si bien el FFM postula que la personalidad se consolida a los 30 años, la trascendencia aumenta con el proceso de envejecimiento, cuando la cercanía con la muerte trae aparejada la necesidad de encontrar un cierre final y un significado para lo vivido (Piedmont, 2005).

De esta manera, las personas mayores pueden haber desarrollado un mayor sentido de trascendencia o, al menos, haber invertido mayor tiempo en su búsqueda (Piedmont, 1999a, 2005). 


\section{Las relaciones causales entre los constructos numinosos y otras variables psicológicas}

De acuerdo con Piedmont (2005), una pregunta relevante acerca de los constructos numinosos radica en si estos pueden o no ser concebidos como predictores de otros constructos (e.g. madurez psicológica).

De esta manera, si la orientación personal a lo numinoso se desarrolla a partir de los rasgos de la personalidad, entonces, las personas con mayor neuroticismo deberían mantener relaciones neuróticas con lo trascendente.

Desde esta perspectiva, la religiosidad y la espiritualidad representarian solamente el reflejo de otros constructos psicológicos, como el de la personalidad (Fredrickson, 2002; Joiner, Perez, \& Walker, 2002).

Para responder a estos interrogantes, a partir de una revisión de diferentes estudios longitudinales, Saroglou (2010) observa que la personalidad resulta predictora de la religiosidad, mientras que ésta no necesariamente afecta los rasgos de la personalidad en casos de conversión religiosa.

Por su parte, Piedmont et al., (2009) examinan diferentes modelos causales para observar (1) si la espiritualidad y la religiosidad constituyen predictores independientes de la madurez psicológica, si son predictores correlacionados, o si deben ser evaluados como un predictor unidimensional y (2) si la religiosidad y la espiritualidad son productos de la madurez psicológica, si la espiritualidad es predictora tanto de la religiosidad como de la madurez psicológica, o si la religiosidad es predictora de la madurez psicológica y de la espiritualidad. 
Los resultados permiten concluir (1) que los constructos numinosos se encuentran altamente correlacionados, pero que no resultan redundantes y (2) que el modelo que mejor se adapta a los datos recabados en campo refleja que la espiritualidad resulta predictora tanto de la madurez psicológica como de la religiosidad.

De acuerdo con Piedmont y Wilkins (2013), estos resultados resultan importantes por dos razones: en primer lugar, sugieren que percibir una relación con una realidad trascendente puede tener consecuencias positivas para la salud emocional de las personas.

En segundo lugar, apoya el modelo de ASPIRES en el que la espiritualidad representa una dimensión motivacional básica que puede o no resultar congruente con el conjunto de rituales y sistemas de creencias que ofrece la religión.

\section{La espiritualidad y la religiosidad como universales}

Si bien algunos trabajos han considerado el papel de la cultura en la relación entre la espiritualidad, la religiosidad y la personalidad (Gebauer et al., 2016; Gebauer, Paulhus, \& Neberich, 2013), en líneas generales, los instrumentos de evaluación reflejan aspectos de la religiosidad y de la espiritualidad propias del cristianismo (e.g., compromiso personal con Jesucristo) y han sido empleadas principalmente en población cristiana (Hill, 2012; Kapuscinski \& Masters, 2010; Moberg, 2002).

Piedmont y Leach (2002) identificaron dos posibles motivos: (1) la mayoría de los investigadores son ellos mismos cristianos y en la mayoría de los estudios su lugar de trabajo son universidades cercanas a la iglesia y (2) las muestras en cristianos son fácilmente accesibles dado que es la fe de mayor presencia en los países donde se llevaron a cabo los estudios. 
Como consecuencia, existe una falta en el pluralismo teológico que podría afectar la posibilidad de desarrollar modelos comprensivos de desarrollo espiritual que presenten validez ecológica.

El desarrollo de ASPIRES surge como un intento de resolver esta cuestión y, como se ha mencionado, ha sido empleado en diferentes contextos como Estados Unidos (Brown, Chen, Gehlert, \& Piedmont, 2013; Jordan, Masters, Hooker, Ruiz, \& Smith, 2014; Piedmont, Wilkins, \& Hollowitz, 2013), China (Chen, 2011), Grecia (Katsogianni \& Kleftaras, 2015), India (Braganza \& Piedmont, 2015), Iran (Ghorbani, Watson, Shamohammadi, \& Cunningham, 2009) y Sri Lanka (Piedmont, Werdel, \& Fernando, 2009).

\section{Discusión}

A partir de los aportes de Gorsuch a mediados de la década de 1980, las publicaciones en el área de la psicología de la religión y de la espiritualidad han crecido exponencialmente.

Como se ha expuesto en este trabajo, numerosos estudios se han ocupado del vínculo entre la religiosidad, la espiritualdiad y la personalidad.

Sin embargo, tal como sugiere Peter Hills, uno de los principales obstáculos para integrar y sistematizar los resultados de las diferentes investigaciones radica en la falta de claridad conceptual en la definición de ambos términos.

Mientras que algunos autores describen los constructos numinosos como orientaciones motivacionales, otros los comprenden como rasgos de la personalidad o estrategias de afrontamiento. 
A la vez, reflejan mayoritariamente las perspectivas teológicas espirituales y religiosas cristianas y occidentales.

La dificultad de los investigadores para tomar distancia de los contextos culturales en donde construyen las herramientas de evaluación parecen limitarlos en la posibilidad de diseñar técnicas capaces de relevar experiencias religiosas y espirituales culturalmente diversas.

Sin embargo, a pesar de la amplia diversidad de técnicas empleadas y de las limitaciones observadas, cabe señalar que se han reportado relaciones significativas entre distintos aspectos ligados a la espiritualidad y la religiosidad y los cinco factores de la personalidad.

En particular, el enofque propuesto por Ralph Piedmont al incluir a la espiritualidad como una sexta dimensión del modelo de los cinco factores de la personalidad parece haber tenido un impacto considerable en la literatura a juzgar por las extensas producciones que lo citan en handbooks y revistas especializadas en el área.

En el mismo sentido, la Escala de Espiritualidad y Sentimientos Religiosos podría representar uno de los insturmentos más empleados debido a que permite distinguir y evaluar diferencialmente la espiritualidad y la religiosidad y a que conceptualizar la espiritualidad como un sexto factor del FFM podría a su vez aportar a esclarecer las relaciones entre los constructos numinosos y otras variables psicológicas, como el bienestar subjetivo y psicológico.

Tal como señalan Ozer y Reise, asociar un constructo al FFM resulta equivalente a establecer la latitud y longitud de una determinada locación en un mapa. En tanto aún resulta necesario precisar, definir y evaluar los constructos numinosos con mayor claridad, su indagación en el 
marco del FFM adquiere particular relevancia para la psicología de la religión y de la espiritualidad.

Financiación: El presente trabajo se enmarca dentro de los proyectos de Investigación UBACyT 20020170200395BA y PICT-2016- 4147 a cargo del Dr. Hugo Simkin acreditados por la Universidad de Buenos Aires (UBA) y la Agencia Nacional de Promoción Científica y Tecnológica (ANPCyT) 


\section{Referencias}

Aghababaei, N. (2012). Religious, honest and humble: Looking for the religious person within the HEXACO model of personality structure. Personality and Individual Differences, 53(7), 880-883. https://doi.org/10.1016/j.paid.2012.07.005

Aghababaei, N. (2013). Between you and God, where is the general factor of personality? Exploring personality-religion relationships in a Muslim context. Personality and Individual Differences, 55(2), 196-198. https://doi.org/10.1016/j.paid.2013.02.021

Aguilar-Vafaie, M. E., \& Moghanloo, M. (2008). Domain and facet personality correlates of religiosity among Iranian college students. Mental Health, Religion \& Culture, 11(5), 461-483. https://doi.org/10.1080/13674670701539114

Ahles, J. J., Mezulis, A. H., \& Hudson, M. R. (2016). Religious coping as a moderator of the relationship between stress and depressive symptoms. Psychology of Religion and Spirituality, 8(3), 228-234. https://doi.org/10.1037/rel0000039

Ahmed, A. M. (2009). Are Religious People More Prosocial? A Quasi-Experimental Study with Madrasah Pupils in a Rural Community in India. Journal for the Scientific Study of Religion, 48(2), 368-374. https://doi.org/10.1111/j.14685906.2009.01452.x

Ahmed, A. M., \& Salas, O. (2013). Religious Context and Prosociality: An Experimental Study from Valparaíso, Chile. Journal for the Scientific Study of Religion, 52(3), 627-637. https://doi.org/10.1111/jssr.12045

Allport, G. W., \& Ross, J. M. (1967). Personal religious orientation and prejudice. Journal of Personality and Social Psychology, 5(4), 432-443. https://doi.org/10.1037/h0021212

Aquino, K., \& Reed, A. (2002). The self-importance of moral identity. Journal of Personality and Social Psychology, 83(6), 1423-1440. https://doi.org/10.1037/0022-3514.83.6.1423

Argyle, M., \& Beit-Hallahmi, Benjamin. (1975). The social psychology of religion. Oxford: Routledge \& Kegan Paul. Retrieved from http:// psycnet.apa.org/psycinfo/1975-29429-000 
Ashton, M. C., \& Lee, K. (2014). Personality and Religiousness. In V. Saroglou (Ed.), Religion, personality, and social behavior (pp. 31-46). New York: Psychology Press.

Azarbaijani, M. (2003). Development of a religious orientation scale based on Islam. Iran: Olive.

Batson, C. D. (1976). Religion as Prosocial: Agent or Double Agent? Journal for the Scientific Study of Religion, 15(1), 29. https://doi.org/10.2307/ 1384312

Batson, C. D., Denton, D. M., \& Vollmecke, J. T. (2008). Quest Religion, AntiFundamentalism, and Limited Versus Universal Compassion. Journal for the Scientific Study of Religion, 47(1), 135-145. https://doi.org/10.1111/j.14685906.2008.00397.x

Batson, C. D., Eidelman, S. H., Higley, S. L., \& Russell, S. A. (2001). "And Who Is My Neighbor?" II: Quest Religion as a Source of Universal Compassion. Journal for the Scientific Study of Religion, 4O(1), 39-50. https://doi.org/10.1111/00218294.00036

Batson, C. D., Floyd, R. B., Meyer, J. M., \& Winner, A. L. (1999). "And Who Is My Neighbor?:" Intrinsic Religion as a Source of Universal Compassion. Journal for the Scientific Study of Religion, 38(4), 445. https://doi.org/10.2307/1387605

Baumeister, R. F., \& Exline, J. (1999). Virtue, Personality, and Social Relations: Self-Control as the Moral Muscle. Journal of Personality, 67(6), 1165-1194. https: / /doi.org/10.1111/1467-6494.00086

Benet-Martinez, V., \& John, O. P. (1998). Los Cinco Grandes across cultures and ethnic groups: multitrait multimethod analyses of the Big Five in Spanish and English. Journal of Personality and Social Psychology, 75(3), 729-50. Retrieved from http:/ /www.ncbi.nlm.nih.gov/pubmed/9781409

Braganza, D., \& Piedmont, R. L. (2015). The Impact of the Core Transformation Process on Spirituality, Symptom Experience, and Psychological Maturity in a Mixed Age Sample in India: A Pilot Study. Journal of Religion and Health, 54(3), 888-902. https://doi.org/10.1007/s10943-015-0049-y

Brown, I. T., Chen, T., Gehlert, N. C., \& Piedmont, R. L. (2013). Age and gender effects on the Assessment of Spirituality and Religious Sentiments (ASPIRES) scale: A cross-sectional analysis. Psychology of Religion and Spirituality, 5(2), 9098. https://doi.org/10.1037/a0030137 
Browne, M., Pennycook, G., Goodwin, B., \& McHenry, M. (2014). Reflective minds and open hearts: Cognitive style and personality predict religiosity and spiritual thinking in a community sample. European Journal of Social Psychology, 44(7), 736-742. https://doi.org/10.1002/ejsp.2059

Bushman, B. J., Ridge, R. D., Das, E., Key, C. W., \& Busath, G. L. (2007). When God Sanctions Killing: Effect of Scriptural Violence on Aggression. Psychological Science, 18(3), 204-207. https://doi.org/10.1111/j.1467-9280.2007.01873.x

Caprara, G. V., Barbaranelli, C., Bermúdez, J., Maslach, C., \& Ruch, W. (2000). Multivariate Methods for the Comparison of Factor Structures in Cross-Cultural Research. Journal of Cross-Cultural Psychology, 31(4), 437-464. https://doi.org/10.1177/0022022100031004002

Carpenter, T. P., \& Marshall, M. A. (2009). An Examination of Religious Priming and Intrinsic Religious Motivation in the Moral Hypocrisy Paradigm. Journal for the Scientific Study of Religion, 48(2), 386-393. https://doi.org/10.1111/j.14685906.2009.01454.x

Chang, E. C., Jilani, Z., Yu, T., Fowler, E. E., Lin, J., Webb, J. R., \& Hirsch, J. K. (2015). Fundamental dimensions of personality underlying spirituality: Further evidence for the construct validity of the RiTE measure of spirituality. Personality and Individual Differences, 75(1), 175-178. https://doi.org/10.1016/j.paid.2014.11.027

Chen, T. P. (2011). A cross-cultural psychometric evaluation of the Assessment of Spirituality and Religious Sentiments Scale in Mainland China. University of Maryland.

Costa, P. T., \& McCrae, R. R. (1992a). Normal personality assessment in clinical practice: The NEO Personality Inventory. Psychological Assessment, 4(1), 5-13. https://doi.org/ 10.1037/1040-3590.4.1.5

Costa, P. T., \& McCrae, R. R. (1992b). Revised NEO Personality Inventory (NEO-PI-R) and NEO Five Factor Inventory (NEO-FFI) Professional Manual. Odessa: PAR.

Costa, P. T., \& McCrae, R. R. (1999). Inventario de Personalidad Neo Revisado (NEO PI-R). Inventario Neo Reducido de Cinco Factores (NEO-FFI). Manual Profesional. Madrid: TEA Ediciones. 
Costa, P. T., McCrae, R. R., \& Dye, D. A. (1991). Facet Scales for Agreeableness and Conscientiousness: A Revision of the NEO Personality Inventory. Personality and Individual Differences, 12(9), 887-898. https://doi.org/10.1016/01918869(91)90177-D

Costa, P. T., McCrae, R. R., \& Norris, A. H. (1981). Personal adjustment to aging: longitudinal prediction from neuroticism and extraversion. Journal of Gerontology, 36(1), 78-85. https://doi.org/10.1093/geronj/36.1.78

Cota-McKinley, A. L., Woody, W. D., \& Bell, P. A. (2001). Vengeance: Effects of gender, age, and religious background. Aggressive Behavior, 27(5), 343-350. https://doi.org/10.1002/ab.1019

Dillon, M., Wink, P., \& Fay, K. (2003). Is Spirituality Detrimental to Generativity? Journal for the Scientific Study of Religion, 42(3), 427-442. https: / /doi.org/10.1111/1468-5906.00192

Dirilen-Gümüş, Ö. (2010). The effect of religiosity on political ideology via value types and personality traits: A comparison between Turkey and USA. Procedia Social and Behavioral Sciences, 5, 12-17. https://doi.org/10.1016/j.sbspro.2010.07.042

Donahue, M. J. (1985). Intrinsic and extrinsic religiousness: Review and metaanalysis. Journal of Personality and Social Psychology, 48(2), 400-419. https: / / doi.org/10.1037/0022-3514.48.2.400

Donnellan, M. B., Oswald, F. L., Baird, B. M., \& Lucas, R. E. (2006). The mini-IPIP scales: tiny-yet-effective measures of the Big Five factors of personality. Psychological Assessment, 18(2), 192-203. https://doi.org/10.1037/10403590.18.2.192

Duck, R. J., \& Hunsberger, B. (1999). Religious Orientation and Prejudice: The Role of Religious Proscription, Right-Wing. International Journal for the Psychology of Religion, 9(3), 157-179. https://doi.org/10.1207/s15327582ijpr0903_1

Duriez, B. (2004). Are religious people nicer people? Taking a closer look at the religion-empathy relationship. Mental Health, Religion \& Culture, 7(3), 249-254. https: / / doi.org/10.1080/13674670310001606450

Duriez, B., Fontaine, J. R. J., Hutsebaut, D., \& Leuven, K. U. (2000). A further elaboration of the post-critical belief scale: Evidence for the existence of four 
different approaches to religion in flanders-belgium. Psychologica Belgica, 40(1967), 153-181.

Duriez, B., \& Hutsebaut, D. (1996). A slow and easy introduction to the Post-Critical Belief. In D. M. Wulff (Ed.), Handbook of the Psychology of Religion and Spirituality. New York: Oxford University Press.

Duriez, B., \& Soenens, B. (2006). Personality, identity styles, and religiosity: An integrative study among late and middle adolescents. Journal of Adolescence, 29(1), 119-135. https://doi.org/10.1016/j.adolescence.2004.11.007

Duriez, B., Soenens, B., \& Beyers, W. (2004). Personality, Identity Styles, and Religiosity: An Integrative Study Among Late Adolescents in Flanders (Belgium). Journal of Personality, 72(5), 877-910. https://doi.org/10.1111/j.00223506.2004.00284.x

Emmons, R. A. (1988). Religion and Personality. In H. G. Koenig (Ed.), Handbook of Religion and Mental Health (pp. 63-74). San Diego: Elsevier.

Emmons, R. A. (1999a). Religion in the Psychology of Personality: An Introduction. Journal of Personality, 67(6), 874-888. https://doi.org/10.1111/14676494.00076

Emmons, R. A. (1999b). The Psychology of Ultimate Concerns: Motivation and Spirituality in Personality. New York: The Guilford Press.

Feagin, J. R. (1964). Prejudice and Relegious Types: A Focused Study of Southern Fundamentalists. Journal for the Scientific Study of Religion, 4(1), 3. https://doi.org/ 10.2307/1385200

Fontaine, J. R. J., Luyten, P., \& Corveleyn, J. (2000). Tell Me What You Believe and I'll Tell You What You Want: Empirical Evidence for Discriminating Value Patterns of Five Types of Religiosity. International Journal for the Psychology of Religion, 10(2), 65-84. https://doi.org/10.1207/S15327582IJPR1002_01

Francis, L. J., \& Bourke, R. (2003). Personality and religion: Applying cattell's model among secondary school pupils. Current Psychology, 22(2), 125-137. https://doi.org/10.1007/s12144-003-1003-9

Francis, L. J., \& Pearson, P. R. (1985). Extraversion and Religiosity. The Journal of Social Psychology, 125(2), 269-270. https://doi.org/10.1080/00224545.1985.9922882 
Francis, L. J., Pearson, P. R., \& Kay, W. K. (1983). Are introverts still more religious? Personality and Individual Differences, 4(2), 211-212. https:/ / doi.org/10.1016/0191-8869(83)90024-7

Fredrickson, B. (2002). How does religion benefit health and well-being? Are positive emotions active ingredients? Psychological Inquiry, 13(3), 209-213. Retrieved from http:/ / www.jstor.org/stable/10.2307/1449332

Gajdosova, B., Orosova, O., Janovska, A., \& Benka, J. (2014). Personality Factors, Autonomy, Religion and Risk Behaviours of First Year Slovak University Students. European Health Psychologist, 17, 726-731.

García, A. M., \& Saroglou, V. (2008). Believing literally versus symbolically : values and personality correlates among Spanish students. Journal of Beliefs \& Values: Studies in Religion \& Education, 29(3), 37-41. https: / /doi.org/10.1080/13617670802465755

Gebauer, J. E., Bleidorn, W., Gosling, S. D., Rentfrow, P. J., Lamb, M. E., \& Potter, J. (2016). Big Five personality and religiosity: Agreeableness and conscientiousness constitute the basis of religiosity only in religious cultures. Journal of Personality and Social Psychology, 42(2).

Gebauer, J. E., Paulhus, D. L., \& Neberich, W. (2013). Big Two Personality and Religiosity Across Cultures: Communals as Religious Conformists and Agentics as Religious Contrarians. Social Psychological and Personality Science, 4(1), $21-$ 30. https://doi.org/10.1177/1948550612442553

Ghorbani, N., Watson, P. J., Shamohammadi, K., \& Cunningham, C. J. (2009). Post-critical beliefs in iran: predicting religious and psychological functioning. Research in the Social Scientific Study of Religion, 20, 151-194.

Goldberg, L. R. (1999). A broad-bandwidth, public domain, personality inventory measuring the lower-level facets of several five-factor models. Personality Psychology in Europe. https://doi.org/citeulike-article-id:1856566

Gosling, S. D., Rentfrow, P. J., \& Swann, W. B. (2003). A very brief measure of the Big-Five personality domains. Journal of Research in Personality, 37(6), 504-528. https://doi.org/10.1016/S0092-6566(03)00046-1

Greenberg, J., Solomon, S., \& Pyszczynski, T. (1997). Terror Management Theory of Self-Esteem and Cultural Worldviews: Empirical Assessments and Conceptual Refinements. In M. P. Zanna (Ed.), Advances in experimental social psychology (pp. 61-139). San Diego: Academic Press. https://doi.org/10.1016/S00652601(08)60016-7 
Greer, T., Berman, M., Varan, V., Bobrycki, L., \& Watson, S. (2005). We Are a Religious People; We Are a Vengeful People. Journal for the Scientific Study of Religion, 44(1), 45-57. https://doi.org/10.1111/j.1468-5906.2005.00264.x

Halama, P., Martos, T., \& Adamovová, L. (2010). Religiosity and Well-Being in Slovak and Hungarian Student Samples: the Role of Personality Traits. Studia Psychologica, 52(2), 111-115.

Heaven, P. C. L., \& Ciarrochi, J. (2007). Personality and religious values among adolescents: A three-wave longitudinal analysis. British Journal of Psychology, 98(4), 681-694. https://doi.org/10.1348/000712607X187777

Henningsgaard, J. M., \& Arnau, R. C. (2008). Relationships between religiosity, spirituality, and personality: A multivariate analysis. Personality and Individual Differences, 45(8), 703-708. https://doi.org/10.1016/j.paid.2008.07.004

Hill, P. C. (2012). Measurement Assessment and Issues in the Psychology of Religion and Spirituality. In R. F. Paloutzian \& C. L. Park (Eds.), Handbook of Psychology of Religion and Spirituality (pp. 48-75). New York: The Guilford Press.

Hoekstra, H. A., Ormel, J., \& De Fruyt, F. (1996). NEO Personality Questionnaires NEO-PI-R, NEO-FFI, Manual. Lisse: Swet \& Zeitlinger BV.

Hunsberger, B. (1995). Religion and Prejudice: The Role of Religious Fundamentalism, Quest, and Right-Wing Authoritarianism. Journal of Social Issues, 51(2), 113-129. https://doi.org/10.1111/j.1540-4560.1995.tb01326.x

James, A., \& Wells, A. (2003). Religion and mental health: towards a cognitivebehavioural framework. British Journal of Health Psychology, 8(3), 359-376. https:/ / doi.org/10.1348/135910703322370905

Joiner, T., Perez, M., \& Walker, R. (2002). Playing devil's advocate: Why not conclude that the relation of religiosity to mental health reduces to mundane mediators? Psychological Inquiry, 13(3), 214-216. Retrieved from http: / / www.jstor.org/stable/ 1449334

Jonathan, E. (2008). The Influence of Religious Fundamentalism, Right-Wing Authoritarianism, and Christian Orthodoxy on Explicit and Implicit Measures of Attitudes Toward Homosexuals. The International Journal for the Psychology of Religion, 18(4), 316-329. https://doi.org/10.1080/10508610802229262 
Jordan, K. D., Masters, K. S., Hooker, S. a., Ruiz, J. M., \& Smith, T. W. (2014). An Interpersonal Approach to Religiousness and Spirituality: Implications for Health and Well-Being. Journal of Personality, 82(5), 418-431. https://doi.org/10.1111/jopy.12072

Kaldestad, E. (1995). The empirical relationships of the religious orientations to personality. Scandinavian Journal of Psychology, 36(1), 95-108.

https://doi.org/10.1111/j.1467-9450.1995.tb00971.x

Kaldestad, E. (1996). The empirical relationships between standardized measures of religiosity and personality/mental health. Scandinavian Journal of Psychology, 37(2), 205-220. https://doi.org/10.1111/j.1467-9450.1996.tb00652.x

Kapuscinski, A. N., \& Masters, K. S. (2010). The current status of measures of spirituality: A critical review of scale development. Psychology of Religion and Spirituality, 2(4), 191-205. https://doi.org/10.1037/a0020498

Katsogianni, I. V., \& Kleftaras, G. (2015). Spirituality, Meaning in Life, and Depressive Symptomatology in Drug Addiction. International Journal of Religion \& Spirituality in Society, 5(2), 11-24.

Kirkpatrick, L. a. (1999). Toward an Evolutionary Psychology of Religion and Personality. Journal of Personality, 67(6), 921-952.

https: / / doi.org/10.1111/1467-6494.00078

Koenig, H. G. (1998). Handbook of religion and mental health. Retrieved from http: / / books.google.com/books?hl=en\&lr=\&id=504GYYj1O3kC\&oi=fnd\&pg=PP2 \&dq=Handbook+of+Religion+and+Mental+Health\&ots=ZUayUs0sgo\&sig=Dgp_uD CHNmi5gqfVCSihHROKDbO

Koenig, H. G., King, D., \& Carson, V. B. (2012). Handbook of religion and health. London: Oxford University Press.

Koenig, H. G., Meador, K. G., \& Parkerson, G. (1997). Religion Index for Psychiatric Research: A 5-Item Measure for Use in Health Outcome Studies. American Journal of Psychiatry, (154), 885-86.

Koenig, H. G., Siegler, I. C., Meador, K. G., \& George, L. K. (1990). Religious coping and personality in later life. International Journal of Geriatric Psychiatry, 5(2), 123-131. https://doi.org/10.1002/gps.930050210 
Krause, N. (2006). Gratitude Toward God, Stress, and Health in Late Life. Research on Aging, 28(2), 163-183. https://doi.org/10.1177/0164027505284048

Leach, M. M., Berman, M. E., \& Eubanks, L. (2008). Religious Activities, Religious Orientation, and Aggressive Behavior. Journal for the Scientific Study of Religion, 47(2), 311-319. https://doi.org/10.1111/j.1468-5906.2008.00409.x

Leak, G. K., \& Fish, S. B. (1999). Development and Initial Validation of a Measure of Religious Maturity. International Journal for the Psychology of Religion, 9(2), 83103. https://doi.org/10.1207/s15327582ijpr0902_1

Lewis, C. A. (1999). An empirical contribution to the psychology of religion: examination of issues in measurement, life-satisfaction and personality theory. University of Ulster.

Lodi-Smith, J., \& Roberts, B. W. (2007). Social Investment and Personality: A MetaAnalysis of the Relationship of Personality Traits to Investment in Work, Family, Religion, and Volunteerism. Personality and Social Psychology Review, 11(1), 6886. https: / / doi.org/10.1177/1088868306294590

MacDonald, D. A. (2000). Spirituality: description, measurement, and relation to the five factor model of personality. Journal of Personality, 68(1), 153-197. https:/ / doi.org/ 10.1111/1467-6494.t01-1-00094

Maclean, A. M., Walker, L. J., \& Matsuba, M. K. (2004). Transcendence and the Moral Self: Identity Integration, Religion, and Moral Life. Journal for the Scientific Study of Religion, 43(3), 429-437. https://doi.org/10.1111/j.14685906.2004.00245.x

Maltby, J., \& Lewis, C. A. (1997). The reliability and validity of a short scale of attitude towards Christianity among USA, English, Republic of Ireland, and Northern Ireland adults. Personality and Individual Differences, 22(5), 649-654. https: / / doi.org/10.1016/S0191-8869(96)00244-9

Maltby, J., Lewis, C. A., Freeman, A., Day, L., Cruise, S. M., \& Breslin, M. J. (2010). Religion and health: the application of a cognitive-behavioural framework. Mental Health, Religion \& Culture, 13(7), 749-759. https://doi.org/10.1080/13674670802596930

McCrae, R. R., \& Costa, P. T. (1999). A five-factor theory of personality. In L. A. Pervin \& O. P. John (Eds.), Handbook of personality: Theory and research (pp. 139-53). New York: The Guilford Press. 
McCrae, R. R., \& Costa, P. T. (2008). Empirical and theoretical status of the fivefactor model of personality traits. In G. J. Boyle, G. Mathews, \& D. H. Saklofske (Eds.), The SAGE Handbook of Personality Theory and Assessment (pp. 273-295). London: Sage Publications.

McCullough, M. E., Enders, C. K., Brion, S. L., \& Jain, A. R. (2005). The Varieties of Religious Development in Adulthood: A Longitudinal Investigation of Religion and Rational Choice. Journal of Personality and Social Psychology, 89(1), 78-89. https: / / doi.org/10.1037/0022-3514.89.1.78

McCullough, M. E., Tsang, J.-A., \& Brion, S. (2003). Personality Traits in Adolescence as Predictors of Religiousness in Early Adulthood: Findings from the Terman Longitudinal Study. Personality and Social Psychology Bulletin, 29(8), 980-991. https://doi.org/10.1177/0146167203253210

McCullough, M. E., \& Willoughby, B. L. B. (2009). Religion, self-regulation, and selfcontrol: Associations, explanations, and implications. Psychological Bulletin, 135(1), 69-93. https://doi.org/10.1037/a0014213

McCullough, M. E., \& Worthington, E. L. (1999). Religion and the Forgiving Personality. Journal of Personality, 67(6), 1141-1164. https: / / doi.org/10.1111/1467-6494.00085

Mirsaleh, Y., Rezai, H., Kivi, S., \& Ghorbani, R. (2010). The role of religiosity, coping strategies, self-efficacy and personality dimensions in the prediction of Iranian undergraduate rehabilitation interns' satisfaction with their clinical experience. Clinical Rehabilitation, 24(12), 1136-1143. https: / / doi.org/ 10.1177/0269215510375907

Moberg, D. O. (2002). Assessing and measuring spirituality: Confronting dilemmas of universal and particular evaluative criteria. Journal of Adult Development, 9(1), 47-60. https://doi.org/10.1023/A:1013877201375

Ostendorf, F., \& Angleitner, A. (2004). NEO-Persönlichkeitsinventar nach Costa und McCrae, revidierte Fassung (NEO-PI-R). Göttingen: Hogrefe.

Ozer, D. J., \& Reise, S. P. (1994). Personality Assessment. Annual Review of Psychology, 45(1), 357-388. https://doi.org/10.1146/annurev.ps.45.020194.002041 
Piedmont, R. L. (1999a). Does Spirituality Represent the Sixth Factor of Personality? Spiritual Transcendence and the Five-Factor Model. Journal of Personality, 67(6), 985-1013. https:/ / doi.org/10.1111/1467-6494.00080

Piedmont, R. L. (1999b). Strategies for using the five-factor model of personality in religious research. Journal of Psychology and Theology, 27(4), 338-350. Retrieved from http://psycnet.apa.org/psycinfo/2000-13311-006

Piedmont, R. L. (2004a). Assessment of spirituality and religious sentiments, technical manual (1st ed.). Timonium, Maryland: Author.

Piedmont, R. L. (2004b). The Logoplex as a paradigm for understanding spiritual transcendence. Research in the Social Scientific Study of Religion, 15(1), 263-284. https://doi.org/10.1037/0893-164X.18.3.213

Piedmont, R. L. (2005). The Role of Personality in Understanding Religious and Spiritual Constructs. In R. F. Paloutzian \& C. L. Park (Eds.), Handbook of the psychology of religion and spirituality (1st ed., pp. 253-273). London: The Guilford Press.

Piedmont, R. L. (2009). The Contribution of Religiousness and Spirituality to Subjective Wellbeing and Satisfaction with Life. In M. Souza, L. J. Francis, J. O'Higgins-Norman, \& D. Scott (Eds.), International Handbook of Education for Spirituality, Care and Wellbeing (Vol. 3, pp. 89-105). Dordrecht: Springer Netherlands. https:/ / doi.org/10.1007/978-1-4020-9018-9

Piedmont, R. L. (2012). Overview and Development of Measure of Numinous Constructs: The Assessment of Spirituality and Religious Sentiments (ASPIRES) Scale. In L. J. Miller (Ed.), The Oxford Handbook of Psychology and Spirituality (pp. 104-122). Oxford: Oxford University Press.

Piedmont, R. L., Ciarrocchi, J. W., Dy-Liacco, G. S., \& Williams, J. E. G. (2009). The empirical and conceptual value of the spiritual transcendence and religious involvement scales for personality research. Psychology of Religion and Spirituality, 1(3), 162-179. https://doi.org/10.1037/a0015883

Piedmont, R. L., \& Leach, M. M. (2002). Cross-Cultural Generalizability of the Spiritual Transcendence Scale in India: Spirituality as a Universal Aspect of Human Experience. American Behavioral Scientist, 45(12), 1888-1901. https: / / doi.org/ 10.1177/0002764202045012011 
Piedmont, R. L., Werdel, M. B., \& Fernando, M. (2009). The utility of the assessment of spirituality and religious sentiments (ASPIRES) scale with Christians and Buddhists in Sri Lanka. Research in the Social Scientific Study of Religion, 2O(1), 131-143.

Piedmont, R. L., \& Wilkins, T. A. (2013). Spirituality, religiousness, and personality: Theoretical foundations and empirical applications. In K. I. Pargament, J. J. Exline, \& J. W. Jones (Eds.), APA handbook of psychology, religion, and spirituality (Vol 1): Context, theory, and research. (Vol. 1, pp. 173-186). Washington: American Psychological Association. https://doi.org/10.1037/14045-009

Piedmont, R. L., Wilkins, T. A., \& Hollowitz, J. (2013). The relevance of spiritual transcendence in a consumer economy: The dollars and sense of it. Journal of Social Research \& Policy, 4(2), 2-19.

Robbins, M., \& Francis, L. J. (2000). Religion, Personality, and Well-being: The Relationship Between Church Attendance and Purpose in Life. Journal of Research on Christian Education, 9(2), 223-238. https: / / doi.org/ 10.1080/10656210009484908

Roberts, B. W., \& Robins, R. W. (2000). Broad Dispositions, Broad Aspirations: The Intersection of Personality Traits and Major Life Goals. Personality and Social Psychology Bulletin, 26(10), 1284-1296. https: / /doi.org/10.1177/0146167200262009

Rose, E. D., \& Exline, J. (2012). Personality, Spirituality, and Religion. In L. J. Miller (Ed.), The Oxford Handbook of Psychology and Spirituality (pp. 85-104). New York: The Guilford Press.

Rowatt, W., \& Kirkpatrick, L. A. (2002). Two Dimensions of Attachment to God and Their Relation to Affect, Religiosity, and Personality Constructs. Journal for the Scientific Study of Religion, 41(4), 637-651. https://doi.org/10.1111/14685906.00143

Salmanpour, H., \& Issazadegan, A. (2012). Religiosity Orientations and Personality Traits with Death Obsession. International Journal of Psychological Studies, 4(1), 150-157. https://doi.org/10.5539/ijps.v4n1p150

Salsman, J. M., Brown, T. L., Brechting, E. H., \& Carlson, C. R. (2005). The link between religion and spirituality and psychological adjustment: the mediating role of optimism and social support. Personality and Social Psychology Bulletin, 31(4), 522-535. https://doi.org/10.1177/0146167204271563 
Sanua, V. D. (1969). Religion, mental health, and personality: a review of empirical studies. American Journal of Psychiatry, 125(9), 1203-1213.

Saroglou, V. (2002). Religion and the five factors of personality: A meta-analytic review. Personality and Individual Differences, 32(1), 15-25. https://doi.org/10.1016/S0191-8869(00)00233-6

Saroglou, V. (2010). Religiousness as a cultural adaptation of basic traits: a fivefactor model perspective. Personality and Social Psychology Review, 14(1), 108125. https: / /doi.org/10.1177/1088868309352322

Saroglou, V., \& Fiasse, L. (2003). Birth order, personality, and religion: a study among young adults from a three-sibling family. Personality and Individual Differences, 35(1), 19-29. https://doi.org/10.1016/S0191-8869(02)00137-X

Saroglou, V., \& Jaspard, J.-M. (2000). Personality and religion: From Eysenck's Taxonomy To The Five-Factor Model. Archive for the Psychology of Religion, 23(1), 41-70.

Saroglou, V., \& Muñoz García, A. (2008). Individual Differences in Religion and Spirituality: An Issue of Personality Traits and/or Values. Journal for the Scientific Study of Religion, 47(1), 83-101. https://doi.org/10.1111/j.14685906.2008.00393.x

Saroglou, V., Pichon, I., Trompette, L., Verschueren, M., \& Dernelle, R. (2005). Prosocial Behavior and Religion: New Evidence Based on Projective Measures and Peer Ratings. Journal for the Scientific Study of Religion, 44(3), 323-348. https://doi.org/10.1111/j.1468-5906.2005.00289.x

Saucier, G., \& Skrzypińska, K. (2006). Spiritual But Not Religious? Evidence for Two Independent Dispositions. Journal of Personality, 74(5), 1257-1292. https://doi.org/10.1111/j.1467-6494.2006.00409.x

Schnell, T. (2012). Spirituality with and without Religion-Differential Relationships with Personality. Archive for the Psychology of Religion, 34(1), 33-61. https://doi.org/10.1163/157361212X644495

Schwartz, S. H., \& Huismans, S. (1995). Value Priorities and Religiosity in Four Western Religions. Social Psychology Quarterly, 58(2), 88. https:/ / doi.org/ 10.2307/2787148 
Shafer, A. B. (1999). Brief Bipolar Markers for the Five Factor Model of Personality. Psychological Reports, (84), 1173-1179.

Simkin, H., \& Azzollini, S. (2015). Personalidad, Autoestima y EspiritualidadReligiosidad desde el Modelo y la Teoría de los Cinco Factores. PSIENCIA: Revista Latinoamericana de Ciencia Psicológica, 7(2), 339-361. https://doi.org/10.5872/psiencia/7.2.22

Streyffeler, L. L., \& McNally, R. J. (1998). Fundamentalists and liberals: personality characteristics of Protestant Christians. Personality and Individual Differences, 24(4), 579-580. https://doi.org/10.1016/S0191-8869(97)00189-X

Taylor, A., \& MacDonald, D. A. (1999). Religion and the five factor model of personality: An exploratory investigation using a Canadian university sample. Personality and Individual Differences, 27(6), 1243-1259. https: / /doi.org/10.1016/S0191-8869(99)00068-9

Tiliopoulos, N., Bikker, A. P., Coxon, A. P. M., \& Hawkin, P. K. (2007). The means and ends of religiosity: A fresh look at Gordon Allport's religious orientation dimensions. Personality and Individual Differences, 42(8), 1609-1620. https: / / doi.org/10.1016/j.paid.2006.10.034

Trapnell, P. D., \& Paulhus, D. L. (2012). Agentic and Communal Values : Their Scope and Measurement. Journal of Personality Assessment, 94(1), 39-52. https: / / doi.org/10.1080/00223891.2011.627968

Village, A. (2011). Outgroup prejudice, personality, and religiosity: Disentangling a complex web of relationships among adolescents in the UK. Psychology of Religion and Spirituality, 3(4), 269-284. https://doi.org/10.1037/a0022966

Walborn, F. (2014). Religion in Personality Theory. London: Elsevier.

Webb, J. R., Toussaint, L., \& Dula, C. S. (2014). Ritualistic, Theistic, and Existential Spirituality: Initial Psychometric Qualities of the RiTE Measure of Spirituality. Journal of Religion and Health, 53(4), 972-985. https://doi.org/10.1007/s 10943013-9697-y

Whitley, B. E. (2009). Religiosity and Attitudes Toward Lesbians and Gay Men: A Meta-Analysis. International Journal for the Psychology of Religion, 19(1), 21-38. https://doi.org/10.1080/10508610802471104 
Willoughby, M. T., Cadigan, R. J., Burchinal, M., \& Skinner, D. (2008). An evaluation of the psychometric properties and criterion validity of the religious social support scale. Journal for the Scientific Study of Religion, 47(1), 147-159. https://doi.org/10.1111/j.1468-5906.2008.00398.x

Wink, P., Ciciolla, L., Dillon, M., \& Tracy, A. (2007). Religiousness, spiritual seeking, and personality: findings from a longitudinal study. Journal of Personality, 75(5), 1051-70. https://doi.org/10.1111/j.1467-6494.2007.00466.x 